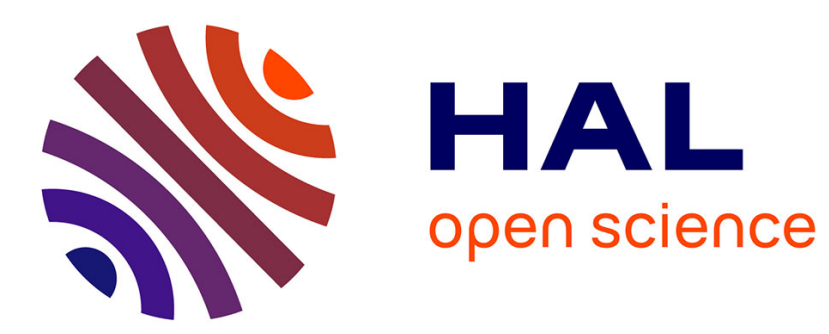

\title{
Functional-integral approach to strongly correlated Fermi systems: Quantum fluctuations beyond the Gutzwiller approximation
}

M. Lavagna

\section{- To cite this version:}

M. Lavagna. Functional-integral approach to strongly correlated Fermi systems: Quantum fluctuations beyond the Gutzwiller approximation. Physical Review B: Condensed Matter and Materials Physics (1998-2015), 1990, 41 (1), pp.142-148. 10.1103/PhysRevB.41.142 . hal-01896293

\section{HAL Id: hal-01896293 \\ https://hal.science/hal-01896293}

Submitted on 16 Oct 2018

HAL is a multi-disciplinary open access archive for the deposit and dissemination of scientific research documents, whether they are published or not. The documents may come from teaching and research institutions in France or abroad, or from public or private research centers.
L'archive ouverte pluridisciplinaire HAL, est destinée au dépôt et à la diffusion de documents scientifiques de niveau recherche, publiés ou non, émanant des établissements d'enseignement et de recherche français ou étrangers, des laboratoires publics ou privés. 


\title{
Functional-integral approach to strongly correlated Fermi systems: Quantum fluctuations beyond the Gutzwiller approximation
}

\author{
M. Lavagna \\ Institut Laue-Langevin, Boíte Postale 156X, 38042 Grenoble CEDEX, France
}

(Received 7 July 1989)

\begin{abstract}
A systematic extension of the model of almost localized fermions to finite temperatures is presented. It consists in accounting for the quantum fluctuations beyond the Gutzwiller approximation (GA). We use the slave-boson formulation of the Hubbard model which has been proved to be equivalent at the saddle-point level to the GA. We determine the values of the Lagrange multipliers introduced to enforce the constraints, and show how they involve a Mott gap at nearly half filling for $U>U_{c}$ (localization edge). The results of the GA for the correlation functions are reproduced at the saddle point when taking into account the implicit dependence of the boson fields with the external excitation. The Gaussian fluctuations are then considered in a renormalized basis of the boson fields which brings out two distinct channels (symmetric $s$ and antisymmetric $a$ ). We show how the free energy and the correlation functions can be simply expressed as a function of the same Landau parameters $F_{0}^{s}$ and $F_{0}^{a}$ defined in the GA.
\end{abstract}

While the prototype of strongly correlated systems is certainly normal ${ }^{3} \mathrm{He},{ }^{1}$ there is now a large class of physical systems that present strong similarities: heavy fermions, ${ }^{2}$ transition-metal oxides, and most likely, the copper oxide superconductors. ${ }^{3}$ In the latter case, it is believed $^{4}$ that the $3 d_{\left(x^{2}-y^{2}\right)}$ orbital of $\mathrm{Cu}$ and the $2 p_{x}, 2 p_{y}$ orbitals of the oxygen hole form a single strongly correlated band. The common features of these systems are a large effective mass, an enhanced magnetic susceptibility, and a reduced compressibility. All these properties reflect the Mott localization due to correlations.

Whereas the week-coupling limit can be attacked by the usual random-phase approximation (RPA), ${ }^{5}$ the strong-correlation regime is more difficult to consider. For instance, there exists no exact solution in dimensionalities $d>1$. In these conditions, the variational ansatz introduced by Gutzwiller ${ }^{6}$ - the Gutzwiller wave function (GWF) - plays a special role, and has been the subject of a great deal of work..$^{7-11}$ Let us just remind the reader that, to make the calculation tractable, one has to introduce the Gutzwiller approximation (GA). This is basically a mean-field approximation which neglects any intersite correlations. It allows one to get an extremely simple formulation of the problem which already brings out the essential physical aspects: ${ }^{10}$ The main one is the localization (or the approach to it) when the system is (or is almost) half filled; the second aspect is the magnetism. The success of the GA is giving a correct description of the Fermi-liquid regime at low temperature, providing a microscopic basis of the Landau theory. On the other hand, it has the usual weaknesses of a mean-field theory: It fails to describe dynamic effects - the dynamic motion of vacancies in the almost localized regime - and has no correct generalization to finite temperature. Unfortunately, within the variational approach, there is no obvious improvement which can be made. In this context, the reformulation of the problem in terms of slave bosons proposed by Kotliar and Ruckenstein ${ }^{12}$ (KR) which reproduced the GA at the saddle-point level, constituted decisive progress since it transforms the variational scheme into a standard pertubative treatment which, in principle, can be pursued beyond the saddle point.

It is essentially the aim of this work to derive the effect of the Gaussian fluctuations on the GA. We would like to mention that several recent papers tackled the same kind of problem in a somewhat different situation. Following the $1 / N$ expansion approach developed in the last two years for heavy fermions, ${ }^{13}$ Kotliar and $\mathrm{Liu}^{14}$ considered the large degeneracy limit of the Hubbard model, and discussed the eventual incidence of the $1 / N$ fluctuations on superconductivity. However, as they pointed out, although the expansion is exact at the order $1 / N$, it does not allow for a description of the magnetism that manifestly occurs in the physical systems closer to the situation $N=2$. Under these conditions, we will consider here the case $N=2$, which is drastically different from a physical point of view, and seems to be more appropriate for describing real systems. We will also quote the recent work of Rasul and $\mathrm{Li},{ }^{15}$ who considered the effect of the fluctuations for the half-filled-band Hubbard model below the localization edge $\left(U<U_{c}\right)$, and obtained some interesting predictions on the $T^{3} \ln T^{3}$ term of the specific heat. They applied it to the case of ${ }^{3} \mathrm{He}$, which appears to be a good candidate for this regime $\left(n=1, U<U_{c}\right)-$ referred to as regime $I$, in the following. The other almost localized regime-referred to as II here-is obtained by introducing some holes from the half-filled strongly correlated case $\left(n \leq 1, U>U_{c}\right)$. It is sensible to think that this "vacancy regime" describes the situation of copper superconductor oxides. It is this regime that we will essentially consider here, with some special emphasis on the analogy with the metallic regime I.

Let us start from the formulation of the Hubbard model introduced in Ref. 12: 


$$
H=\sum_{i j \sigma} t_{i j} \bar{z}_{i \sigma}^{\dagger} \bar{z}_{j \sigma} c_{i \sigma}^{\dagger} c_{j \sigma}+U \sum_{i} d_{i}^{\dagger} d_{i}-\sum_{i \sigma}(\mu+\sigma h) n_{i \sigma}
$$

with

$$
\bar{z}_{i \sigma}=e_{i}^{\dagger} p_{i \sigma}+p_{i-\sigma}^{\dagger} d_{i},
$$

where $t_{i j}$ and $U$ are respectively, the nearest-neighbor hopping and the on-site Coulomb repulsion between electrons of different spins, $c_{i \sigma}^{\dagger}\left(c_{i \sigma}\right)$ are creation (annihilation) operators for an electron of $\operatorname{spin} \sigma(= \pm 1)$ at site $i$, and $\mu$ its chemical potential; and $e_{i}, p_{i \sigma}$, and $d_{i}$, auxiliary boson fields such as $e_{i}^{\dagger} e_{i}, p_{i \sigma}^{\dagger} p_{i \sigma}$, and $d_{i}^{\dagger} d_{i}$, represent the projectors on the empty, singly occupied $\sigma$, and doubly occupied $i$ site. Equation (1a) is strictly equivalent to the Hubbard model as soon as the following constraints are satisfied:

$$
\begin{aligned}
& P_{i}=e_{i}^{\dagger} e_{i}+\sum_{\sigma} p_{i \sigma}^{\dagger} p_{i \sigma}+d_{i}^{\dagger} d_{i}-1=0, \\
& Q_{i \sigma}=c_{i \sigma}^{\dagger} c_{i \sigma}-p_{i \sigma}^{\dagger} p_{i \sigma}-d_{i}^{\dagger} d_{i}=0 \forall \sigma .
\end{aligned}
$$

Since the time evolution generated by $H$ preserves the constraints $\left(\left[H, P_{i}\right]=\left[H, Q_{i \sigma}\right]=0\right)$, it is only necessary to enforce the constraints at one particular time; this is done by introducing time-independent Lagrange multipliers $\lambda_{i}^{(1)}$ and $\lambda_{i \sigma}^{(2)}$. Before going further, let us remark that the choice of $\bar{z}_{i \sigma}$ is not unique, and one can replace ${ }^{11,12} \bar{z}_{i \sigma}$ by any combination $U_{i \sigma} \bar{z}_{i \sigma} V_{i \sigma}$, where $U_{i \sigma}$ and $V_{i \sigma}$ are diagonal operators such as $U_{i \sigma}=1$ in the $(0)$ and $(-\sigma)$ configurations, and $V_{i \sigma}=1$ in $(\sigma)$ and $(\uparrow \downarrow)$. One can in particular choose, as did KR, ${ }^{12}$

$z_{i \sigma}=\left(1-d_{i}^{\dagger} d_{i}-p_{i \sigma}^{\dagger} p_{i \sigma}\right)^{-1 / 2} \bar{z}_{i \sigma}\left(1-e_{i}^{\dagger} e_{i}-p_{i-\sigma}^{\dagger} p_{i-\sigma}\right)^{-1 / 2}$.

All the choices are formally equivalent as far as the constraints are exactly satisfied at each site and any time. This is no longer true when one makes approximations. Typically, the mean-field approximation satisfies the constraint on average, and leads to different results depending on the special choice of $z_{i \sigma}$. These discrepancies are supposed to disappear if one could include quantum fluctuations at all orders. When stopping at mean field, the choice (3) is more sensible since it renormalizes $\bar{z}_{i \sigma}$ by its value in the uncorrelated case, and gives back the free electron gas in the limit $U=0$. It is this choice that will be retained in all the following.

The partition function $Z$ can be written as a functional integral over the fermion and boson fields

$$
Z=\int \mathscr{D} c \mathscr{D} e \mathcal{D} p_{\sigma} \mathscr{D} d \prod_{i \sigma} d \lambda_{i}^{(1)} d \lambda_{i \sigma}^{(2)} \exp \left[-\int_{0}^{\beta} \mathcal{L}(\tau) d \tau\right] \text {, }
$$

where the Lagrangian $\mathcal{L}(\tau)$ is

$$
\begin{aligned}
\mathcal{L}(\tau)= & \sum_{i, j, \sigma} c_{i \sigma}^{\dagger}\left[\left(\partial_{\tau}-\mu-\sigma h+\lambda_{i \sigma}^{(2)}\right) \delta_{i j}+z_{i \sigma}^{\dagger} z_{j \sigma} t_{i j}\right] c_{j \sigma}+\sum_{i} e_{i}^{\dagger}\left(\partial_{\tau}+\lambda_{i}^{(1)}\right) e_{i} \\
& +\sum_{i, \sigma} p_{i \sigma}^{\dagger}\left(\partial_{\tau}+\lambda_{i}^{(1)}-\lambda_{i \sigma}^{(2)}\right) p_{i \sigma}+\sum_{i} d_{i}^{\dagger}\left(\partial_{\tau}+\lambda_{i}^{(1)}-\sum_{\sigma} \lambda_{i \sigma}^{(2)}+U\right) d_{i} .
\end{aligned}
$$

At the saddle point (uniform and static boson fields), one can integrate over the Grassman variables and obtain for the free energy

$$
\begin{aligned}
F_{0}= & f_{0}+U d_{0}^{2}+\lambda_{0}^{(1)}\left(e_{0}^{2}+\sum_{\sigma} p_{0 \sigma}^{2}+d_{0}^{2}-1\right) \\
& +\sum_{\sigma} \lambda_{0 \sigma}^{(2)}\left(n_{\sigma}-p_{0 \sigma}^{2}-d_{0}^{2}\right)-\sum_{\sigma}(\mu+\sigma h) n_{\sigma},
\end{aligned}
$$

where

$$
f_{0}=-\frac{1}{\beta} \sum_{k, i \omega_{n}, \sigma} \ln \left(-i \omega_{n}+q_{0 \sigma} \varepsilon_{k}\right)
$$

in the notation

$$
\begin{aligned}
& q_{0 \sigma}=z_{0 \sigma}^{2}, \\
& \varepsilon_{k}=\sum_{i j} t_{i j} \exp \left[i \mathbf{k} \cdot\left(\mathbf{R}_{i}-\mathbf{R}_{j}\right)\right] .
\end{aligned}
$$

It appears then clearly that the saddle point of the slaveboson formulation of the Hubbard model is equivalent to the Gutzwiller approximation. ${ }^{12}$

The saddle-point equations are obtained by minimizing the free energy $F_{0}$ with respect to the seven parameters $e_{0}, p_{0 \sigma}, d_{0}, \lambda_{0}^{(1)}$, and $\lambda_{0 \sigma}^{(2)}$. This leads, in addition to three constraints, to the following equations in the paramagnetic case (where the 0 indices are now omitted):

$$
\begin{aligned}
& \lambda^{(1)}=\frac{4 p^{2}}{1-\delta^{2}} \frac{x}{e}|\varepsilon|\left(1+\frac{2 e x}{1-\delta}\right), \\
& U+\lambda^{(1)}-2 \lambda^{(2)}=\frac{4 p^{2}}{1-\delta^{2}} \frac{x}{d}|\varepsilon|\left(1+\frac{2 d x}{1+\delta}\right), \\
& \lambda^{(1)}-\lambda^{(2)}=2 x^{2}|\varepsilon|\left(1+\frac{2 p^{2}}{1-\delta}+\frac{2 p^{2}}{1+\delta}\right),
\end{aligned}
$$

where $|\varepsilon|$ is the averaged energy by site in the uncorrelated case

$$
|\varepsilon|=2 \int \omega \rho_{0}(\omega) d \omega, \quad \delta=1-n,
$$

and where we have used the convenient notations introduced by Vollhardt et al: ${ }^{9} \quad x=e+d$. Let us recall that, in this notation, $e, p, d$ (and so $q$ ) can be written 


$$
\begin{aligned}
& d^{2}=\left[\frac{x^{2}-\delta}{2 x}\right]^{2}, \\
& e^{2}=\left[\frac{x^{2}+\delta}{2 x}\right]^{2}, \\
& p^{2}=\frac{2 x^{2}-x^{4}-\delta^{2}}{4 x^{2}}, \\
& q=\frac{2 x^{2}-x^{4}-\delta^{2}}{1-\delta^{2}} .
\end{aligned}
$$

By combining (6a), (6b), and (6c), one can eliminate the Lagrange multipliers, and obtain the usual optimization equation of the Gutzwiller approximation. This result is not surprising since it simply means

$$
d\left(f_{0}+U d_{0}^{2}\right) / d d_{0}^{2}=0
$$

where $e_{0}$ and $p_{0}$ are expressed as a function of $d_{0}$ using the constraints. The resulting equation at $T=0$ is the following:

$$
\left(1-x^{2}\right) \frac{x^{4}}{x^{4}-\delta^{2}}=\tilde{u}
$$

with

$$
\widetilde{u}=u \frac{1-\delta^{2}}{1-\alpha \delta^{2}}
$$

and

$$
u=\frac{U}{8\left|\varepsilon_{0}\right|},
$$

where $\left|\varepsilon_{0}\right|$ is the value of the energy $|\varepsilon|$ at half filling. One can in principle solve the system of equations (8) at any point of the diagram $(U, \delta)$. In fact, we will content ourselves with describing the most interesting region (in the since of the correlations) in the proximity of halffilling. A glance at the behavior of

$$
A(x)=\left(1-x^{2}\right) x^{4} /\left(x^{4}-\delta^{2}\right)
$$

enables us to distinguish two regimes:

Regime I (metallic regime) (Refs. 6 and 7), $\tilde{u} \simeq U<1$, $n=1$. Then

$$
\begin{aligned}
& x^{2}=1-u, \\
& e^{2}=d^{2}=(1-u) / 4, \\
& p^{2}=(1+u) / 4, \\
& q=1-u^{2}
\end{aligned}
$$

Regime II (vacancy regime) (Ref. 9), $\tilde{u}=u>1$, $x \simeq \sqrt{\delta} \ll 1$. Then

$$
\begin{aligned}
& x^{2}=\delta / \zeta \\
& e^{2}=\frac{\delta(1+\zeta)^{2}}{4 \zeta} \\
& d^{2}=\frac{\delta(1-\zeta)^{2}}{4 \zeta} \\
& p^{2}=\frac{1}{2} \\
& q=2 \delta / \zeta
\end{aligned}
$$

with

$$
\zeta=\sqrt{1-U_{c} / U}
$$

One recovers the Brinkman-Rice ${ }^{7}$ transition (Mott localization) above a critical edge of localization $U_{c}=8\left|\varepsilon_{0}\right|$. Above, one needs to introduce some vacancies (or electrons) to restore the metallic behavior. This is the conduction by vacancies. Note that, for infinite $U$, double occupation is strictly forbidden $(d=0)$, while the bandwidth $\sim \delta$ is different from zero in the vacancy regime.

Indeed, the content of the saddle-point equations (6) goes beyond the Gutzwiller approximation, and also determines the values of the Lagrange multipliers at the saddle point. One finds the following.

Regime I:

$$
\begin{aligned}
& \lambda^{(1)}=\frac{U_{c}}{4}(1+u)(2-u), \\
& \lambda^{(2)}=\frac{U}{2} .
\end{aligned}
$$

\section{Regime II:}

$$
\begin{aligned}
& \lambda^{(1)}=\frac{U}{2}(1 \pm \xi)\left[1+\frac{\delta}{\zeta}(1+\xi)\right], \\
& \lambda^{(2)}=\frac{U}{2}(1 \pm \xi)\left[1-\frac{\delta}{2 \zeta}(1+\xi)\right] .
\end{aligned}
$$

( - for $n \leq 1$ and + for $n \geq 1$ ). The variation of $\lambda^{(1)}$ and $\lambda^{(2)}$ as a function of $U$ is reported in Fig. 1. Note the opening of a gap delimited by the values of $\lambda^{(2)}$ in the vacancy regime $(n \leq 1)$ and the electronic regime $(n \geq 1)$. In fact, one can demonstrate the formal identity between the Lagrange multiplier $\lambda^{(2)}$ and the chemical potential $\mu$ derived from $(-d F / d \delta)$. The observed gap of width $\Delta=U \sqrt{1-U_{c} / U}$ (Fig. 1) is nothing else but the Mott gap. While $\lambda^{(1)}$ and $\lambda^{(2)}$ notably differ in regime $I$, their being closer (within $\delta$ ) in regime II is a clear manifestation which may take a physical meaning not yet understood. Table I summarizes the saddle-point values of the boson fields and Lagrange multipliers in regimes $I$ and II (up to corrections in $\delta^{2}$ ).

Before studying the quantum fluctuations around the Gutzwiller approximation, we will end this discussion on the saddle point by considering the linear response of the system to an external excitation. It is suitable to distinguish whether one works at fixed (independent of the external excitation) or variable (with an implicit dependence of the boson fields with with the external excitation) boson fields. Typically, the first procedure is 


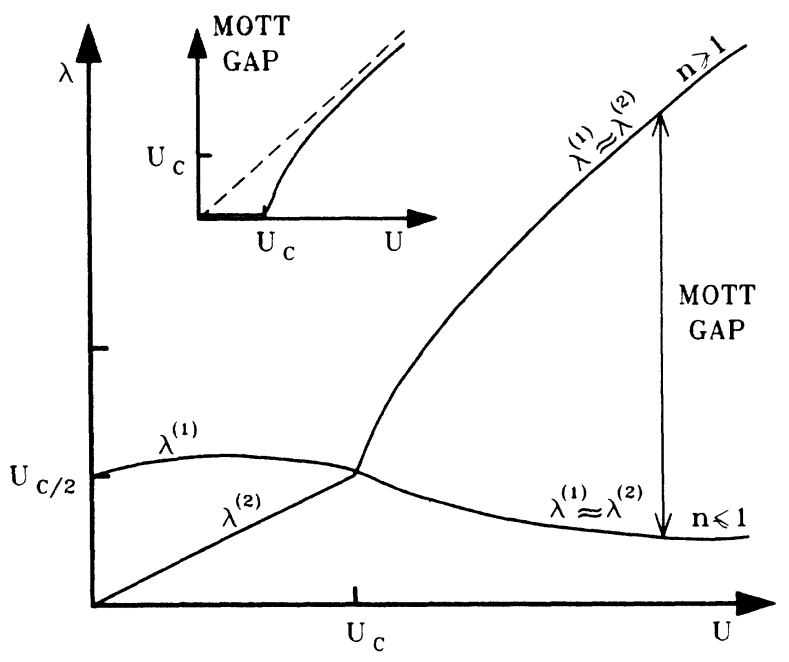

FIG. 1. Saddle-point values of the Lagrange multipliers $\lambda^{(1)}$ and $\lambda^{(2)}$ as functions of $U$. Notice the opening of a Mott gap for $U>U_{c}$. In the insert, $U$ dependence of the Mott gap.

ate the contribution of an elementary fermion loop

$\frac{1}{\beta} \sum_{\mathbf{k}, \omega_{n}} G\left(\mathbf{k}, \omega_{n}\right) \boldsymbol{G}\left(\mathbf{k}+\mathbf{q}, \omega_{n}+\omega\right)=\chi_{0}\left(\mathbf{q} / k_{F}, \omega / E_{F}^{*}\right) q_{0}^{-1}$,

where $\chi_{0}$ is the correlation function for the free-electron gas, and $E_{F}^{*}=k_{F} / 2 m^{*}$ is related to the effective mass $m^{*}=m q_{0}^{-1}$. On the other hand, the evaluation of the correlation functions requires the implementation of the second procedure. The correlation functions (density density, spin spin, density spin) derived from the expansion of the free energy (as a function of $\delta^{2}, M^{2}, \delta M$ ) show an enhancement factor due to the implicit dependence of $q_{\sigma}$ with $\delta$ and $M$, and lead, in Fermi-liquid language, to $F_{0}^{s}$ and $F_{0}^{a}$ different from zero. In other words, this is equivalent to considering the saddle point in the presence of an external field. This situation is to be linked to the Stoner model in itinerant magnetism (weak- $U$ limit of the Hubbard model), where the contribution to the elementary fermion loop is simply $\chi_{0}\left(\mathbf{q} / k_{F}, \omega / E_{F}\right)$, while the correlation functions drawn from the expansion of the free energy

$$
\{U n \uparrow n \downarrow=U[(n+M-\delta) / 2][(n-M-\delta) / 2]\}
$$

present an enhancement factor: $\kappa(0,0)=\chi_{0} /\left(1+U \chi_{0}\right)$, $\chi_{s}(0,0)=\chi_{0} /\left(1-U \chi_{0}\right)$, and $\chi_{c}(0,0)=0$. The Stoner theory already gives the structure of linear response that is obtained further by considering the fluctuations in the RPA. The situation here is similar, and one finds for the susceptibility

$$
\chi_{s}(0,0)=\chi_{0} q_{0}^{-1} /\left(1-\frac{\left.\frac{\partial\left(\sum_{\sigma} q_{\sigma}\right)}{\partial\left(\boldsymbol{M}^{2}\right)}\right|_{d}}{2 \alpha q_{0}}\right),
$$

where $\alpha$ is a parameter that characterizes the initial band of density $\rho_{0}: \alpha=1 /\left(4 \rho_{0}|\varepsilon|\right)=1$ for a rectangular bond and $3 \pi^{2} / 32=0.925$ for an elliptic one. Expression (14) has been obtained by noting that $\left(\Sigma_{\sigma} q_{\sigma}\right)$ is quadratic in $M$, and the saddle-point position ensures $d F / d M^{2}=\partial F /$ $\left.\partial M^{2}\right|_{d}$. An explicit calculation gives the general expression

TABLE I. Saddle-point values of boson fields and Lagrange multipliers in regimes I and II (within the notation $\zeta=\sqrt{1-1 / u}$ ).

\begin{tabular}{lcc}
\hline \hline & Regime I $\left(n=1, U<U_{c}\right)$ & Regime II $\left(n \lesssim 1, U>U_{c}\right)$ \\
\hline$x^{2}$ & $(1-u)+\left(\frac{u}{(1-u)^{2}}-u(\alpha-1)\right] \delta^{2}$ & $\frac{\delta}{\zeta}-\frac{\delta^{2}}{2 u \xi^{4}}$ \\
$e^{2}$ & $\frac{(1-u)}{4}+\frac{\delta}{2}+\left(\frac{1}{4(1-u)^{2}}-\frac{u(\alpha-1)}{4}\right) \delta^{2}$ & $\frac{\delta}{4 \zeta}(1+\zeta)^{2}-\frac{\delta^{2}}{8 u^{2} \zeta^{4}}$ \\
$d^{2}$ & $\frac{(1-u)}{4}-\frac{\delta}{2}+\left(\frac{1}{4(1-u)^{2}}-\frac{u(\alpha-1)}{4}\right) \delta^{2}$ & $\frac{\delta}{4 \zeta}(1-\zeta)^{2}-\frac{\delta^{2}}{8 u^{2} \xi^{4}}$ \\
$p^{2}$ & $\frac{(1+u)}{4}-\left(\frac{1}{4(1-u)^{2}}-\frac{u(\alpha-1)}{4}\right) \delta^{2}$ & $\frac{1}{2}-\frac{\left(1+\zeta^{2}\right)}{4 \zeta} \delta+\frac{\delta^{2}}{\left.8 u^{2} \xi^{4}\right)}$ \\
$q$ & $\frac{\left(1-u^{2}\right)+\left(\frac{2 u^{2}}{(1-u)^{2}}-1-2 u^{2}(\alpha-1)\right.}{\left(1-\delta^{2}\right)}$ & $\frac{2 \delta}{\zeta}-\left(1+\frac{1}{\zeta^{4}}\right) \delta^{2}$ \\
$\lambda^{(1)}$ & $\frac{U_{c}}{4}(1+u)(2-u)$ & $\frac{U}{2}(1-\zeta)\left(1+\frac{\delta}{\zeta}(1+\zeta)\right)$ \\
$\lambda^{(2)}$ & $\frac{U}{2}$ & $\frac{U}{2}(1-\zeta)\left(1-\frac{\delta}{2 \zeta}(1+\zeta)\right)$ \\
$\lambda^{(1)}-\lambda^{(2)}$ & $\frac{U_{c}}{4}(1-u)(2+u)$ & $\frac{3 U_{c}}{4} \frac{\delta}{\zeta}$ \\
\hline \hline
\end{tabular}




$$
\chi_{s}(0,0)=\frac{\chi_{0} q_{0}^{-1}}{1-\frac{1}{\alpha}\left[1-\frac{e d}{(e+d)^{2}} \frac{1}{\left(2 p^{2}\right)^{2}}\right]}
$$

One recovers

$$
F_{0}^{a}=\left[-1+1 /(1+u)^{2}\right] \alpha^{-1}
$$

in regime $I$ and

$$
F_{0}^{a}=[-1+1 / 4 u] \alpha^{-1}
$$

in regime II. In the same way, one finds

$$
\kappa(0,0)=\chi_{0} q_{0}^{-1} /\left(1-\frac{\frac{d}{d \delta}\left[\left.\frac{\partial q}{\partial \delta}\right|_{d}\right]}{2 \alpha q_{0}}\right) .
$$

Note $^{16}$ that the denominator cannot simplify as for the susceptibility since $q$ has a linear term in $\delta$. One recovers the results given in Refs. 9 and 11 , respectively, as follows.

Regime I:

$$
\left.\frac{1}{2 \kappa}=q_{0}\left|\varepsilon_{0}\right|(\alpha-1)+\frac{1}{(1-u)^{2}}\right],
$$

i.e.,

$$
F_{0}^{s}=\left[-1+1 /(1-u)^{2}\right] \alpha^{-1} .
$$

Regime II:

$$
\frac{1}{2 \kappa}=\left|\varepsilon_{0}\right|\left(1+q_{0}(\alpha-1)+\frac{1}{\zeta^{2}}\right),
$$

i.e.,

$$
F_{0}^{s}=\left(-1+\frac{2 u-1}{2 \delta \sqrt{u(u-1)}}\right) \alpha^{-1} .
$$

On the other hand, the free energy has no mixed term in $M \delta$, and as in any RPA theory, $\chi_{c}(0,0)=0$. As for the Stoner theory, one expects that the consideration of Gaussian fluctuations enables us to confirm the structure of the correlation functions obtained at $q=\omega=0$ in the Gutzwiller approximation (saddle point with "variable" slave-boson fields). We will see in the following that this is effectively the case.

For the consideration of quantum fluctuations, it is useful to remark as $\operatorname{Rasul}$ and $\mathrm{Li}^{15}$ that $\mathcal{L}(\tau)$ obeys a local gauge invariance [cf. Eq. (4b)]:

$$
\begin{aligned}
& e_{i} \rightarrow \exp \left(i \theta_{i}\right) e_{i}^{\prime}, \\
& d_{i} \rightarrow \exp \left[i\left(\theta_{i}-\sum_{\sigma} \chi_{i \sigma}\right)\right] d_{i}^{\prime}, \\
& p_{i \sigma} \rightarrow \exp ^{i\left(\theta_{i}-\chi_{i \sigma}{ }^{\prime}\right.} p_{i \sigma}, \\
& c_{i \sigma} \rightarrow \exp ^{i \chi_{i \sigma}} c_{i \sigma}, \\
& \lambda_{i}^{(1)} \rightarrow \lambda_{i}^{(1)^{\prime}}+i \dot{\theta}_{i}, \\
& \lambda_{i \sigma}^{(2)} \rightarrow \lambda_{i \sigma}^{(2)^{\prime}}+i \dot{\chi}_{i \sigma} .
\end{aligned}
$$

It is often more convenient to absorb the phases of the boson fields into the Lagrange multipliers which turn out to also be fields. This defines the "radial" gauge that will be retained in the following since it introduces only real fields. After slightly modifying the writing of the constraints

$$
\lambda_{i}^{(1)} P_{i}+\sum_{\sigma} \lambda_{i \sigma}^{(2)} Q_{i \sigma}=\lambda_{i}^{(1)} P_{i}+\frac{1}{2}\left(\sum_{\sigma} \lambda_{i \sigma}^{(2)}\right)\left(\sum_{\sigma} Q_{i \sigma}\right)+\frac{1}{2}\left(\lambda_{i \uparrow}^{(2)}-\lambda_{i \downarrow}^{(2)}\right)\left(Q_{i \uparrow}-Q_{i \downarrow}\right)
$$

we define the renormalized boson fields

$$
\alpha_{i}=\left\{\alpha_{i}, i=1,7\right\}=\left\{e, d, \frac{p_{\uparrow}+p_{\downarrow}}{2}, \lambda^{(1)}, \frac{\lambda_{\uparrow}^{(2)}+\lambda_{\downarrow}^{(2)}}{2}, \frac{p_{\uparrow}-p_{\downarrow}}{2}, \frac{\lambda_{\uparrow}^{(2)}-\lambda_{\downarrow}^{(2)}}{2}\right\} .
$$

The basis $\left\{\alpha_{i}\right\}$ has the advantage of making the unperturbated boson propagator $\widetilde{D}_{0}^{-1}$ block diagonal in the paramagnetic case,

$$
\widetilde{D}_{0}^{-1}=\left[\begin{array}{cc}
\widetilde{D}_{0 s}^{-1} & 0 \\
0 & \widetilde{D}_{0 a}^{-1}
\end{array}\right]
$$

where

$$
\widetilde{D}_{0 s}^{-1}=\left[\begin{array}{ccccc}
\lambda_{0}^{(1)} & 0 & 0 & e_{0} & 0 \\
0 & U+\lambda_{0}^{(1)}-2 \lambda_{0}^{(2)} & 0 & d_{0} & -2 d_{0} \\
0 & 0 & 2\left(\lambda_{0}^{(1)}-\lambda_{0}^{(2)}\right) & 2 p_{0} & -2 p_{0} \\
e_{0} & d_{0} & 2 p_{0} & 0 & 0 \\
0 & -2 d_{0} & -2 p_{0} & 0 & 0
\end{array}\right]
$$

and 


$$
\widetilde{D}_{0 a}^{-1}=\left[\begin{array}{cc}
2\left(\lambda_{0}^{(1)}-\lambda_{0}^{(2)}\right) & -4 p_{0} \\
-4 p_{0} & 0
\end{array}\right]
$$

We will see that the block $(s)$ connected to $\left(\sum_{\sigma} n_{\sigma}\right)$ is related to the density-density correlations, and the block $(a)$ connected to $\left(n_{\uparrow}-n_{\downarrow}\right)$ is related to the spin-spin correlations. The zero off-diagonal terms ensure $\chi_{c}=0$. The analogy with the Stoner theory is here again obvious $\left(U n_{\uparrow} n_{\downarrow}=U\left(n_{\uparrow}+n_{\downarrow}\right)^{2} / 4-U\left(n_{\uparrow}-n_{\downarrow}\right)^{2} / 4\right)$, giving $F_{0}^{s}=+U$ and $F_{0}^{a}=-U$. Including the fluctuations, the Lagrangian can be written

$$
\left.\mathcal{L}(\tau)=\sum_{i, j, \sigma} c_{i \sigma}^{\dagger}\left[\partial_{\tau}+\mid z_{0 \sigma}^{\dagger}+\delta z_{i \sigma}^{\dagger}\right)\left(z_{0 \sigma}+\delta z_{j \sigma}\right) t_{i j}+\left(\lambda_{0 \sigma}^{(2)}+\delta \lambda_{i}^{(2)}\right) \delta_{i j}\right] c_{j \sigma}+\sum_{\mathbf{q}} \alpha_{\mathbf{q}}^{\dagger} \widetilde{D}_{0}^{-1} \boldsymbol{\alpha}_{\mathbf{q}} .
$$

Let us note $\mathcal{L}_{1}(\tau)$ the first term of the Lagrangian

$$
\mathcal{L}_{1}(\tau)=\sum_{\mathbf{k}, \mathbf{q}, \sigma} c_{\mathbf{k}+\mathbf{q}, \sigma}^{\dagger}\left[\left(\partial_{\tau}+q_{0 \sigma} \varepsilon_{k}+\lambda_{0}^{(2)}\right) \delta_{q}+z_{0} \delta z_{-\mathbf{q}, \sigma}^{\dagger} \varepsilon_{k}+z_{0} \delta z_{\mathbf{q}, \sigma} \varepsilon_{k+q}+\sum_{\kappa} \delta z_{\kappa, \sigma}^{\dagger} \delta z_{\kappa+\mathbf{q}, \sigma} \varepsilon_{k+\kappa+q}+\delta \lambda_{\mathbf{q}, \sigma}\right] c_{\mathbf{k} \sigma}
$$

By expanding to the first 2 orders in $\alpha$ :

$$
\begin{aligned}
\mathcal{L}_{1}(\tau)=\sum_{\substack{\mathbf{k}, \mathbf{q}, \sigma \\
\alpha_{i}=\alpha_{i}^{\dagger}, \alpha_{i} \\
i=1 \text { to }}} c_{\mathbf{k}+\mathbf{q}, \sigma}^{\dagger}\{ & \left(\partial_{\tau}+q_{0 \sigma} \varepsilon_{k}+\lambda_{0}^{(2)}\right) \delta_{q}+z_{0} \varepsilon_{k}\left[\frac{\partial z_{\sigma}^{\dagger}}{\partial \alpha_{i}} \bar{\alpha}_{i}(-\mathbf{q})+\frac{\partial^{2} z_{\sigma}^{\dagger}}{\partial \bar{\alpha}_{i} \partial \bar{\alpha}_{j}} \bar{\alpha}_{i}(-\mathbf{q}) \bar{\alpha}_{j}(-\mathbf{q})\right] \\
& +z_{0} \varepsilon_{k+q}\left[\frac{\partial z_{\sigma}}{\partial \alpha_{i}} \bar{\alpha}_{i}(\mathbf{q})+\frac{\partial^{2} z_{\sigma}}{\partial \bar{\alpha}_{i} \partial \bar{\alpha}_{j}} \bar{\alpha}_{i}(\mathbf{q}) \bar{\alpha}_{j}(\mathbf{q})\right] \\
& \left.+\left[\sum_{\kappa} \varepsilon_{k+\kappa+q}\left[\frac{\partial z_{\sigma}^{\dagger}}{\partial \bar{\alpha}_{i}} \frac{\partial z_{\sigma}}{\partial \bar{\alpha}_{j}} \bar{\alpha}_{i}(\kappa) \bar{\alpha}_{j}(\boldsymbol{\kappa}+\mathbf{q})\right]\right]+\frac{\partial \lambda_{\sigma}^{(2)}}{\partial \bar{\alpha}_{i}} \bar{\alpha}_{i}(\mathbf{q})\right\} c_{\mathbf{k} \sigma} .
\end{aligned}
$$

By integrating over the Grassman variables, and expanding up to the second order in the boson variables, ${ }^{17}$ one obtains the Gaussian corrections $S^{(2)}$ to the saddle-point action $S^{(0)}$ :

$$
\begin{aligned}
S^{(2)} & =\frac{1}{\beta} \sum_{\substack{\mathbf{q} \\
i=1 \text { to } 7}} \alpha_{i}^{\dagger}(\mathbf{q})\left[\left(\widetilde{D}_{0}^{-1}\right)_{i j}+\sum_{\mathbf{k}, \sigma} A_{i j}^{(\sigma)}(\mathbf{k}, \mathbf{q}) G_{\mathbf{k} \sigma}+\sum_{\mathbf{k}, \sigma} B_{i}^{\sigma *}(\mathbf{k}, \mathbf{q}) B_{j}^{\sigma}(\mathbf{k}, \mathbf{q}) G_{\mathbf{k} \sigma} G_{\mathbf{k}+\mathbf{q}, \sigma}\right] \alpha_{j}(\mathbf{q}) \\
& =\frac{1}{\beta} \sum_{\mathbf{q}} \boldsymbol{\alpha}^{\dagger}(\mathbf{q}) \widetilde{D}(\mathbf{q}) \boldsymbol{\alpha}(\mathbf{q}),
\end{aligned}
$$

(where the notation $\mathbf{k}$ is used for $\mathbf{k}$ vector and frequency)

$$
\begin{aligned}
A_{i j}^{\sigma}(\mathbf{k}, \mathbf{q})= & z_{0} \varepsilon_{k} \frac{\partial^{2}\left(z_{\sigma}^{\dagger}+z_{\sigma}\right)}{\partial \alpha_{i}^{\dagger} \partial \alpha_{j}} \delta_{\mathbf{q}}+\varepsilon_{k+q}\left(\frac{\partial z_{\sigma}^{\dagger}}{\partial \alpha_{i}^{\dagger}} \frac{\partial z_{\sigma}}{\partial \alpha_{j}}+\frac{\partial z_{\sigma}}{\partial \alpha_{i}^{\dagger}} \frac{\partial z_{\sigma}^{\dagger}}{\partial \alpha_{j}}\right] \text { if } i, j=1,2,3, \text { or } 6 \\
& =0 \text { if } i, j=4,5 \text {, or } 7 \\
B_{i}^{\sigma(*)}(\mathbf{k}, \mathbf{q}) & =z_{0}\left[\varepsilon_{k} \frac{\partial z_{\sigma}^{\dagger}}{\partial \alpha_{i}^{(\dagger)}}+\varepsilon_{k+q} \frac{\partial z_{\sigma}}{\partial \alpha_{i}^{(\dagger)}}\right] \text { if } i, j=1,2,3 \text {, or } 6 \\
& =\frac{\partial \lambda_{\sigma}^{(2)}}{\partial \alpha_{i}^{(\dagger)}} \text { if } i=5 \text { or } 7 \\
& =0 \text { if } i=4 .
\end{aligned}
$$

By summing on the bosonic variables, one gets the contribution to the free energy

$$
F^{(2)}=\frac{1}{\beta} \sum_{\mathbf{q}, \omega_{v}} \ln \left[\operatorname{det} \widetilde{D}^{-1}\left(\mathbf{q}, \omega_{v}\right)\right]
$$

In the (sensible) approximation where one can neglect the $\mathbf{k}$ dependence of the coefficient $B_{i}, \widetilde{D}^{-1}$ can be written in the form

$$
\widetilde{D}^{-1}\left(\mathbf{q}, \omega_{v}\right)=\widetilde{D}_{1}^{-1}+\widetilde{\Gamma} \chi_{0}\left(\mathbf{q}, \omega_{v}\right),
$$

where in the basis $\left\{\alpha_{i}\right\}, \widetilde{D}_{1}^{-1}$ and $\widetilde{\Gamma}$ are both block diago- nal. When only the linear terms in $\chi_{0}$ are kept (RPA):

$$
\begin{aligned}
F^{(2)}= & \frac{1}{\beta} \sum_{\mathbf{q}, \omega_{v}} \ln \left[1+\operatorname{Tr}\left(\widetilde{D}_{1 s} \widetilde{\Gamma}_{s}\right) \chi_{0}\left(\mathbf{q}, \omega_{v}\right)\right] \\
& +\ln \left[1+\operatorname{Tr}\left(\widetilde{D}_{1 a} \widetilde{\Gamma}_{a}\right) \chi_{0}\left(\mathbf{q}, \omega_{v}\right)\right] .
\end{aligned}
$$

We have calculated analytically $\operatorname{Tr}\left(\widetilde{D}_{1 s} \widetilde{\Gamma}_{1 s}\right)$ and $\operatorname{Tr}\left(\widetilde{D}_{1 a} \widetilde{\Gamma}_{1 a}\right)$. It is remarkable that the expressions found for these two terms in regimes I and II coincides with the coefficients $\left(-F_{0}^{s}\right)$ and $\left(-F_{0}^{a}\right)$ obtained in a much simpler way through equations (16) and (18) (within a $\chi_{0}^{-1}$ factor). 
The effect of the fluctuations not only confirms the structure obtained at $\mathbf{q}=\omega=0$, but also provides the dynamic aspect which was missing in the Gutzwiller approximation. Equation (31) allows for a direct evaluation of the free energy at finite temperature. This avoids the delicate question of determining the entropy in the Gutzwiller approximation. Let us mention that a naive mapping of the entropy on the ideal-gas case would have given a $R \ln 4$ entropy term at high temperature in contradiction with the almost localized picture where the entropy is bounded by $R \ln 2$ on a large plateau $T_{F}^{*} \leq T \leq U$ before reaching the entropy of the ideal gas at higher temperature. This was the motivation of the astucious extension proposed by Seiler et $a l .{ }^{8}$ that remains essentially phenomenological. The direct evaluation of the entropy is possible here, and work is currently in progress in this direction. On the other hand, one can easily deduce the dynamic correlation functions

$$
\begin{aligned}
& \kappa(\mathbf{q}, \omega)=\frac{\chi_{0}(\mathbf{q}, \omega) q_{0}^{-1}}{1+F_{0}^{s} \chi_{0}(\mathbf{q}, \omega) / \chi_{0}}, \\
& \chi_{s}(\mathbf{q}, \omega)=\frac{\chi_{0}(\mathbf{q}, \omega) q_{0}^{-1}}{1+F_{0}^{a} \chi_{0}(\mathbf{q}, \omega) / \chi_{0}}, \\
& \chi_{c}(\mathbf{q}, \omega)=0 .
\end{aligned}
$$

In regime $I$ and in the weak $U$ limit, Eq. (16) and (18) gives $F_{0}^{s}=2 U$ and $F_{0}^{a}=-2 U$ allowing us to find from Eq. (30) the RPA results of weak coupling. In regime II, $F_{0}^{s} \sim 1 / \delta$ and the compressibility $\kappa$ is found to be unenhanced as expected for an incompressible Fermi liquid.

The discussion of the eventual instabilities (magnetic, flux phase, ${ }^{18}$ superconducting . ...) essentially depends on the structure of the correlation function $\chi_{0}$ considered in the unperturbated case. For a spherical symmetry, $\chi_{0}$ is given by the Lindhard functions, and one finds a ferromagnetic instability at large $U$ but no antiferromagnetic instability. The situation is rather different in the case of an alternated structure (e.g. cubic) where the nesting property of the paramagnetic Fermi surface at half filling gives an AF instability at $n=1$ for infinitesimal small value of $U$. The corresponding phase diagram is reported in Ref. 12. It is easy to see that the ferromagnetic instability occurs above $U_{F}=1 /[4(1-\alpha)]$ at $n=1$. The $F$ AF boundary is asymptotic to the line $n=1$ at $U=\infty$ as required by Nagaoka's theorem. ${ }^{19}$

From a general point of view, the Fermi surface of the perturbated system is identical to that of the unperturbated system. Then, the eventuality of a superconducting or flux phase might be examined by already breaking the symmetry at the unperturbated level (through $\chi_{0}$ ). In that sense, we think that the above calculation may be useful since it may be adapted to any "unperturbated" structure. It constitutes a frame in which a set of parameters $q_{0}=m / m^{*}, F_{0}^{s}, F_{0}^{a}, \lambda_{0}^{(1)}, \lambda_{0}^{(2)}$ are defined independently of the structure. It would be interesting to apply it to some more complex situations (flux phase, superconductivity ...) perhaps in closer connection to the physics of high- $T_{c}$ superconductors.

\section{ACKNOWLEDGMENTS}

I am grateful to Professor Nozieres for his comments and encouragement during the course of this work. It is a pleasure to thank T. Dombre, P. Grange, D. Grempel, J. Rodriguez, and N. Schopohl for stimulating and useful discussions.
${ }^{1}$ P. W. Anderson and W. Brinkman, The Physics of Liquid and Solid Helium, Part II, edited by K. H. Bennemann and J. B. Ketterson (Wiley, New York, 1978).

${ }^{2}$ P. A. Lee, T. M. Rice, J. W. Serene, L. J. Sham, and J. W. Wilkins, Comments Solid State Phys. 12, 99 (1986).

${ }^{3} \mathrm{P}$. W. Anderson, in Frontiers and Borderlines in Many Body Physics (North-Holland, Amsterdam, 1987); P. W. Anderson, Science 235, 1196 (1987); G. Baskaran, Z. Zou, and P. W. Anderson, Solid State Commun 63, 973 (1987).

${ }^{4}$ F. C. Zhang and T. M. Rice, Phys. Rev. B 37, 3759 (1988).

${ }^{5}$ N. F. Berk and J. R. Schrieffer, Phys. Rev. Lett. 17, 433 (1966); S. Doniach and S. Engelsberg, ibid. 17, 7502 (1966).

${ }^{6}$ M. C. Gutzwiller, Phys. Rev. Lett. 10, 159 (1963); Phys. Rev. 134, A923 (1964); 137, A1726 (1965).

${ }^{7}$ W. F. Brinkman and T. M. Rice, Phys. Rev. B 2, 4302 (1970).

${ }^{8}$ K. Seiler, C. Gros, T. M. Rice, K. Ueda, and D. Vollhardt, J. Low Temp. Phys. 64, 195 (1986).

${ }^{9}$ D. Vollhardt, P. Wolfe, and P. W. Anderson, Phys. Rev. B 35, 6703 (1987).

${ }^{10}$ D. Vollhardt, Rev. Mod. Phys. 56, 99 (1984).

${ }^{11}$ P. Nozieres (unpublished).

${ }^{12}$ G. Kotliar and A. E. Ruckenstein, Phys. Rev. Lett. 57, 1362 (1986).

${ }^{13}$ A. J. Millis and P. A. Lee, Phys. Rev. B 35, 3394 (1987); A.
Auerbach, and K. Levin, Phys. Rev. Lett. 57, 877 (1986); M. Lavagna, A. J. Millis, and P. A. Lee, ibid. 58, 266 (1987).

${ }^{14}$ G. Kotliar and J. Liu, Phys. Rev. Lett. 61, 1784 (1988).

15 J. W. Rasul and T. Li, J. Phys. C 21, 5119 (1988).

${ }^{16}$ Note that, since $q$ possesses a linear term in $\delta$,

$$
\frac{d}{d \delta}\left[\left.\frac{\partial q}{\partial \delta}\right|_{d}\right]=\left.\frac{\partial^{2} q}{\partial \delta^{2}}\right|_{d}+\frac{\partial}{\partial d^{2}}\left[\left.\frac{\partial q}{\partial \delta}\right|_{d}\right] \frac{d d^{2}}{d \delta}
$$

is not equivalent to $\partial^{2} q /\left.\partial \delta^{2}\right|_{d}$, contrary to the case of the susceptibility [Eq. (14)]. Note too that one could also derive the compressibility by working not at $d=c^{\text {ste }}$ but at $x=c^{\text {st }}$, or also by explicitly taking account of the dependence of the free energy with $\delta$.

${ }^{17}$ We only keep the contribution of the term $\alpha_{i}^{\dagger} \alpha_{i}$ and neglect for the moment that of the anomalous terms $\alpha_{i}^{\dagger} \alpha_{j}^{\dagger}$ (or $\alpha_{i} \alpha_{j}$ ).

${ }^{18}$ For the $\frac{1}{2}$ flux phase, see, e.g., G. Kotliar, Phys. Rev. B 37, 3664 (1988); I. Affleck and J. B. Marston, ibid. 37, 3774 (1988). For the generalized flux phase, see P. W. Anderson, B. S. Shastry, and D. Hristopoulos (unpublished); P. Lederer, D. Poilblanc, and T. M. Rice (unpublished).

${ }^{19}$ Y. Nagaoka, Phys. Rev. 147, 392 (1966). 